\title{
Elemental surface analysis at ambient pressure by electron-induced $x$-ray fluorescence
}

\author{
Jason E. Feldman, Jaroslava Z. Wilcox, a) and Thomas George \\ Jet Propulsion Laboratory, 4800 Oak Grove Drive, Pasadena, California 91109 \\ David N. Barsic and Axel Scherer \\ California Institute of Technology, 1200 E. California Boulevard, Pasadena, California 91125
}

(Received 5 June 2000; accepted 16 October 2002)

\begin{abstract}
The development of a portable surface elemental analysis tool, based on the excitation of characteristic $\mathrm{x}$ rays from samples at ambient pressure with a focused electron beam is described. This instrument relies on the use of a thin electron transmissive membrane to isolate the vacuum of the electron source from the ambient atmosphere. The major attributes of this instrument include rapid (several minutes) spectrum acquisition, nondestructive evaluation of elemental composition, no sample preparation, and high-to-medium (several hundreds $\mu \mathrm{m}$ ) spatial resolution. The instrument proof-of-principle has been demonstrated in a laboratory setup by obtaining energy dispersive x-ray spectra from metal and mineral samples. (c) 2003 American Institute of Physics.
\end{abstract}

[DOI: $10.1063 / 1.1542889]$

\section{INTRODUCTION}

Rapid and nondestructive analyses of surface elemental composition in atmosphere can provide extremely useful information in the fields of mineralogy, materials science, and biology. Three excitation sources currently used for x-ray fluorescence analysis of surface composition in ambient pressure are: (i) a radioactive alpha particle source, (ii) an x-ray source, or (iii) a focused ion beam. Here we present the possibility of using a fourth technique, the excitation of characteristic x-ray fluorescence using a focused electron beam, which we have no demonstrated in ambient atmosphere. Electron beam excited energy dispersive $\mathrm{x}$-ray analysis (EDAX or EDX) is a widely accepted technique for determining the elemental surface composition of samples in vacuum, for example, in analytical scanning electron microscopy (SEM). It has, however, not been previously demonstrated in ambient atmosphere due to the difficulty of generating and transmitting electron beams through the atmosphere. In this work we present a proof-of-principle for a new instrument, the atmospheric electron x-ray spectrometer (AEXS). The AEXS is a novel instrument concept based on the idea of using a thin electron-transmissive membrane to encapsulate an electron source thus isolating the vacuum within the source from the ambient pressure, yet letting the electrons transmit into the outside environment. ${ }^{1-3}$ The AEXS electron source consists of an electron emitter and an electrostatic column that focuses and accelerates the electrons. When used with a short membrane-to-sample working distance, samples in the ambient atmosphere can be irradiated using the focused electron beam. The transmitted beam through the membrane impinges on the external sample generating characteristic $\mathrm{x}$ rays. The $\mathrm{x}$ rays are collected and

${ }^{a)}$ Electronic mail: jaroslava.z.wilcox@jpl.nasa.gov analyzed using an energy dispersive detector to perform elemental composition analysis for the irradiated spot.

Two methods are currently available for the analysis of samples at atmospheric pressures using electron beams. The method on which the AEXS is based utilizes a thin electrontransparent membrane strong enough to support a differential pressure of $1 \mathrm{~atm}$ differential and maintain a high vacuum within an electron column. The use of a vacuum-isolation membrane has been advocated in the past for the construction of an atmospheric SEM, ${ }^{1,3}$ and has been implemented to construct a device used for electron beam charging of small particles and subsequent electrostatic deflection. ${ }^{2}$ An alternative method for analysis at near-ambient pressure is the environmental SEM $\left(\mathrm{ESEM}^{4}\right)$, which employs a series of pressure-limiting apertures with pumping stages to isolate the electron source vacuum from the pressure in the sample chamber. Both approaches provide rapid analysis times, small size for the irradiated spot, and evaluation of both insulating and conducting samples is possible due to the charge neutralization provided by ionized ambient gas interacting with charges on the sample surfaces. The reason why we have investigated the membrane encapsulation approach is because we expect it to result in a more compact instrument suitable for field deployment. ${ }^{5,6}$ Although the ESEM permits a smaller spot size than the AEXS, the requirement for active vacuum pumping of multiple stages is expected to result in a much more elaborate vacuum system and hence a much less portable instrument. Energy-dispersive x-ray detectors determine the height of current pulses resulting from the fluorescent $\mathrm{x}$ rays emitted from the interrogated samples by using a low-noise $p$ - $n$ diode. Recently, it has become possible to miniaturize these detector systems without significantly compromising their energy resolution, as the size of cryogenic cooling systems has been miniaturized. Thus the concept of a portable microprobe to perform surface elemental composition analysis at ambient pressure is realistic. 




FIG. 1. The AEXS instrument concept with the major components: encapsulated electron source with a membrane, power supply, and x-ray detector system.

The proof-of-principle of the AEXS has been demonstrated at our laboratory at JPL using a laboratory setup with a thermionic electron source to generate the electrons. ${ }^{6}$ So far, with the electrostatic acceleration up to $10 \mathrm{keV}$ and a 200-nm-thick silicon nitride ( $\mathrm{SiN}$ ) membrane window, we have performed surface elemental analysis for external samples at $1 \mathrm{~atm}$ pressure with the irradiated spot diameter approximately $0.8 \mathrm{~mm}$. The $\mathrm{x}$ rays were detected by a cooled silicon $p$ type-intrinsic- $n$ type $(p-i-n)$ diode $\mathrm{x}$-ray detector. The electron induced $\mathrm{x}$-ray generation is very efficient, using a $10 \mu \mathrm{A}$ beam current the analysis time was as short as 100 s. Theoretical models predict further improvements for the performance of an optimized instrument. ${ }^{5}$

\section{AEXS ELECTRON SOURCE}

The AEXS concept is shown schematically in Fig. 1. The principle components of the instrument are the miniature electron source, the electron-transmissive vacuum isolation membrane, the high power supply and electronics system, and the energy dispersive $\mathrm{x}$-ray detector. The proof-ofprinciple for the AEXS has been demonstrated by using a laboratory (breadboard) apparatus that consists of the same components as in Fig. 1, with the addition of a turbomolecular high vacuum pump to maintain the vacuum enclosure pressure at approximately $10^{-6}$ Torr. A high vacuum gate valve separated the vacuum chamber from the membrane mounting chamber, allowing for separate purging and pumping of the membrane chamber, thereby allowing rapid exchange of the membrane without damaging the electron gun assembly. The electron source was a commercially available Sony electron tube (Song pat No. FM2210), capable of operating at $12 \mathrm{kV}$, and supplied by CRT Scientific Corporation (Van Nuys, CA). The electronics included a high voltage power supply for the electron gun, a shaping amplifier, and a silicon $p-i$ - $n$ diode $\mathrm{x}$-ray detector cooled to $255 \mathrm{~K}$. The electron tube consists of a thermionic filament to generate the electrons, electrostatic focusing electrodes, and an acceleration anode. The cathode, extraction, and focusing electrodes were operated at $-9.9,-9.72$, and $-10.23 \mathrm{kV}$, respectively, with the anode grounded. The emitted electrons are accelerated in the electrostatic column and are focused through the membrane window onto a sample at 1 atm pressure, where they generate characteristic $\mathrm{x}$ rays for elemental composition analysis of the irradiated spot.

\section{ELECTRON TRANSMISSIVE MEMBRANE}

The electron gun is mounted in a vacuum enclosure that is isolated from the ambient atmosphere by a thin electrontransmissive membrane. The membrane was low-stress 200nm-thick SiN. The membrane was fabricated by using low pressure chemical vapor deposition of a 200-nm-thick pinhole-free SiN film on both sides of a 400- $\mu$ m-thick silicon wafer. One side of the wafer was coated with photoresist, and $1.5 \times 1.5 \mathrm{~mm}^{2}$ openings were created using optical photolithography. The SiN film within these openings was removed with reactive ion etching. The photoresist was removed and the wafer was back-etched in potassium hydroxide $(\mathrm{KOH})$ up to the etch-resistant top SiN film, leaving a pyramidal-shaped etch-resistant SiN-covered silicon support frame for mechanical strength. The frame was attached to a stainless steel flange using Epotech (Epoxy Technology, MA) H20E silver epoxy. The final membrane size was $1 \times 1 \mathrm{~mm}^{2}$.

The membrane eliminates the need to introduce the investigated sample into an evacuated chamber as is done in SEM. Because of its critical role, the membrane properties were investigated prior to the incorporation into the laboratory setup. The membrane must be sufficiently thin to transmit the electron beam, yet it must be mechanically robust to withstand the differential pressure to isolate the vacuum of the electron column from the ambient atmosphere. The membrane properties were investigated both theoretically and experimentally through a series of tests that determined that a 1 $\mathrm{mm}^{2}$ membrane is capable of isolating high vacuum, able to withstand differential pressure in excess of 1 atm, survives vibrational shocks, and has high electron transmissivity.

The electron transmissivity was modeled using Monte Carlo simulations and was determined experimentally using measurements in both the SEM and the laboratory setups. Attenuation and beam spreading occur due to the interaction of electrons with the intervening membrane and atmosphere molecules. Figure 2 shows the predicted ${ }^{5}$ transmissivity and beam spreading due to electron scattering events in the membrane and the external atmosphere as a function of the working distance between the membrane and the target at $1 \mathrm{~atm}$ pressure for a 200 -nm-thick $\mathrm{SiN}$ membrane. The simulations were performed using the conventional formulas ${ }^{7}$ for interaction of high-energy charged particles with matter, with corrections added to those formulas following the experimental results obtained with low energy electron beams in electronbeam lithography. ${ }^{8}$ The transmissivity and beam spreading depend on electron energy, membrane composition and thickness, and atmosphere. For example, for $10 \mathrm{keV}$ electrons at a $1 \mathrm{~mm}$ working distance at 1 atm pressure, the predicted transmissivity in Fig. 2 is $80 \%$ and the irradiated spot diameter is $0.6 \mathrm{~mm}$, whereas at $2 \mathrm{~mm}$ working distance the predicted diameter is $1 \mathrm{~mm}$. Here, the spot size was defined as the arithmetic average of distances from the beam center at which the electrons impinge on the sample. Note that within the experimental error, the measured spot size at a $2 \mathrm{~mm}$ working distance in the laboratory setup agrees with theoretical predictions.

In addition to experiments in the AEXS test setup, the 


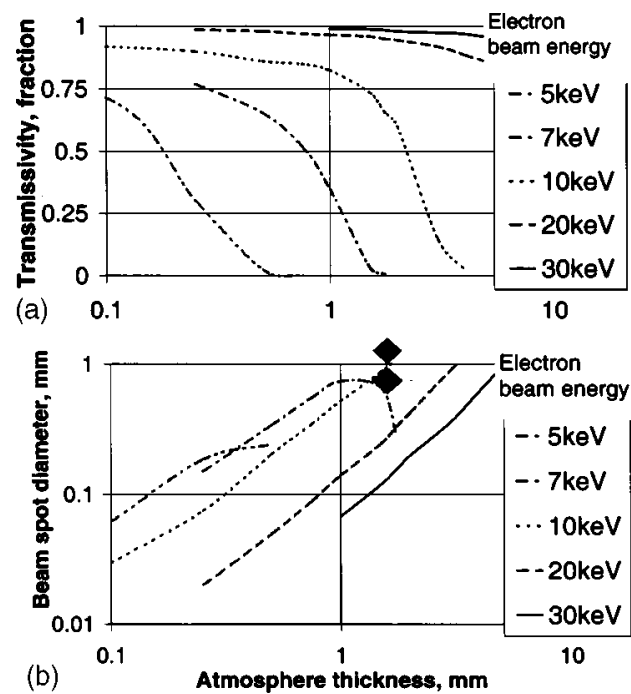

FIG. 2. Predicted electron (a) transmissivity and (b) spot size as a function of the working distance at $1 \mathrm{~atm}$ pressure for a 200 -nm-thick SiN membrane. Note that the measured spot size ( $\boldsymbol{\Lambda}$ designates experimental data taken at $10 \mathrm{keV}$ and $2 \mathrm{~mm}$ working distance) agrees with the predicted spot size within the experimental error.

membranes were subjected to and successfully withstood vibration and shock tests designed for the engineering qualification of the Mars'01 lander at the JPL test bed. The diffusion of air through the membrane was also measured in a separate apparatus to determine the leak rate that would eventually degrade the vacuum in the electron column. The rate of pressure increase in a $5 \mathrm{~cm}^{3}$ volume isolated by a 2.25 $\mathrm{mm}^{2}$ area, 200-nm-thick membrane was found to be very low, about $2 \times 10^{-5} \mathrm{Torr} / \mathrm{yr}$.

\section{DATA ACQUISITION}

Electron transmission through the membrane was first confirmed by introducing a sample coated with a $\mathrm{ZnO}$ phosphor into the path of the electron beam. A significant reduction in the phosphorescence was observed when a 5- $\mu \mathrm{m}$ thick polycarbonate film was introduced between the membrane and the phosphor. Following this preliminary observation, we proceeded to obtain $\mathrm{x}$-ray spectra from metals. To verify that the observed fluorescence was indeed excited by the electrons, a $5-\mu \mathrm{m}$-thick polycarbonate film was inserted between the membrane and the target, resulting in a strong reduction of the characteristic x-ray signal. When, however, the polycarbonate film was inserted between the target and the $\mathrm{x}$-ray detector, only very slight reduction was observed. These results confirm that the excitation particles are electrons.

The irradiated spot size was roughly determined by observing the luminous spot on the phosphor screen. A diameter between 0.5 and $1.5 \mathrm{~mm}$ was observed at a $2 \mathrm{~mm}$ working distance. It should be noted that both electrons and $\mathrm{x}$ rays can excite phosphorescence. Due to the inevitable presence of spurious $\mathrm{x}$ rays, the measured diameter provides us with an upper limit to the electron spot size. These experiments also showed that the $1 \mathrm{~mm}^{2}$ area, 200-nm-thick SiN membranes are capable of withstanding a differential pressure of $1 \mathrm{~atm}$.
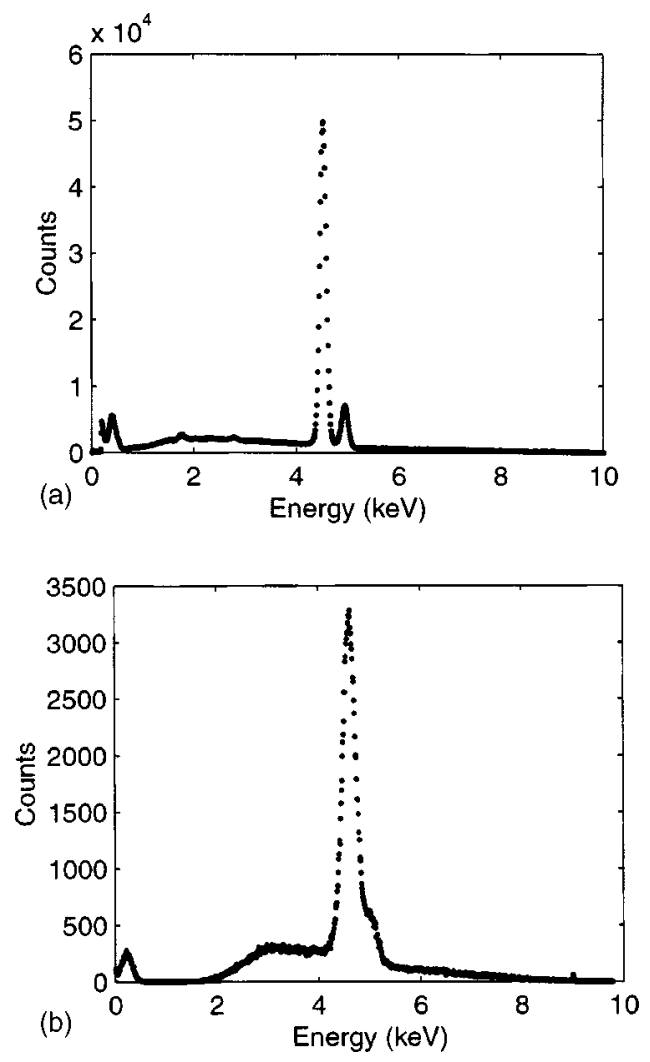

FIG. 3. Acquired x-ray spectrum from an irradiated Ti sample by a $10 \mathrm{keV}$ electron beam in (a) SEM, and (b) laboratory AEXS. Since the beam current was not measured, the number of counts for SEM and AEXS measurements has not been correlated. The particular spectrum acquisition time in the AEXS was $1000 \mathrm{~s}$; however, similar spectra were obtained with times as short as $100 \mathrm{~s}$.

The $\mathrm{x}$ rays were detected by a $\mathrm{Si}-p-i-n$ photodiode based system developed by Amptek (Amptek Inc., Bedford, MA, detector part No. XR-100CR) with a 4- $\mu$ m-thick beryllium window, a PX2CR amplifier, and cooled using a Peltier cooler. This system has a spectral resolution between $165 \mathrm{eV}$ (24 $\mu$ s dead time) and $200 \mathrm{eV}$ (12 $\mu$ s dead time) full width at half maximum, which is somewhat better than the XR-100T system that was flown on Mars Pathfinder. ${ }^{9}$ The detector was calibrated by using an Am x-ray fluorescing source. X-ray spectra from this target were collected with a commercial Kevex EDX system, and compared with the AEXS spectra. The comparison was used to identify known spectral peaks, and these peaks were used to calibrate the energy scale of the acquired data.

The calibrated system was used to acquire $\mathrm{x}$-ray data for several metal and mineral samples in the outside atmosphere. The spectra (about 10000 data points) acquisition time was typically several minutes, and analysis times as short as 100 $\mathrm{s}$ were obtained for about $10 \mu \mathrm{A}$ beam current. One of the samples was $99.99 \%$ pure Ti mechanically polished with 800 grit sandpaper. The sample was placed $2 \mathrm{~mm}$ from the membrane in the outside atmosphere. The collected $\mathrm{x}$-ray spectrum is compared to the spectrum obtained by using a SEMmounted EDX analysis system in Figs. 3(a) and 3(b). Figure 3(a) shows the spectrum taken in the SEM, and Fig. 3(b) shows that taken with the AEXS setup. Note that the two clearly discernible peaks (Ti $K \alpha$ and Ti $K \beta$ ) in Fig. 3(a) are 
observed as a single, slightly shifted peak with a small shoulder in Fig. 3(b). The peaks are more resolved in the SEM due to a greater detector resolution. Whereas the SEM detector is cooled to $77 \mathrm{~K}$, the AEXS detector is cooled only to $255 \mathrm{~K}$. The observed increase in the background level [Fig. 3(b)] at about $2.5 \mathrm{keV}$ just beyond the $\mathrm{Si} K \alpha$ energy is believed to be associated with $\mathrm{X}$-ray fluorescence of the $\mathrm{SiN}$ window.

\section{DISCUSSION}

In conclusion, by using a laboratory setup, we have demonstrated rapid acquisition of spectrally and spatially resolved x-ray spectra excited by a high-energy electron beam that has been transmitted through a thin $(200 \mathrm{~nm})$ vacuumisolation $\mathrm{SiN}$ membrane, for samples at $1 \mathrm{~atm}$ pressure. By using a $10 \mathrm{keV}$ electron beam and a $2 \mathrm{~mm}$ working distance, the irradiated spot size was between 0.5 and $1.5 \mathrm{~mm}$, and the analysis time was as short as $100 \mathrm{~s}$. Thus the AEXS, while eliminating the need to introduce the sample into an evacuated chamber, retains most of the essential chemical analysis features of a laboratory SEM. We expect this new analysis tool to find its place among other ambient pressure surface analysis systems. While other portable instruments, such as $\mathrm{X}$-ray fluorescence spectrometers (XRD Corp., Somerville, MA), analyze surface regions of dimensions of several square centimeters, techniques such as external micro ionbeam analysis $\left(\mathrm{XMIBA}^{10}\right)$, external nuclear reaction analysis (XNRS), and external Rutherford backscattering spectrometer (XRBS) provide focused beams and spatial resolution as small as several square $\mu \mathrm{m}$ but require large stationary instruments. The portability and medium resolution of the AEXS fills the gap between nonportable, high-resolution laboratory instruments and portable, low-resolution in situ instruments.

For samples in Earth atmosphere, a $10 \mathrm{keV}$ beam will attenuate significantly when the working distance exceeds about $5 \mathrm{~mm}$ (Fig. 2). Figure 2 also shows that the electron transmissivity and spatial resolution increase by reducing the working distance and by increasing the electron energy; the figures of merit also improve by working in reduced atmospheres such as on Mars (approximately 7 Torr). While Fig. 2 predicts that by using a $25 \mathrm{keV}$ beam it will be possible to resolve areas several hundreds of $\mu \mathrm{m}$ in diameter, Monte Carlo simulations performed for Martian atmosphere predicted resolved areas as small as $100 \mu \mathrm{m}$ at a $2 \mathrm{~mm}$ working distance. ${ }^{5}$ The AEXS laboratory setup was built around a turbopumped vacuum system with the objective to demonstrate the feasibility of energy-dispersive analysis using an electron beam transmitted through a thin membrane. A more compact system is currently undergoing development as a step towards a miniature instrument. Presently we believe that the overall size of a miniature instrument will be limited by the size of a miniature high voltage power supply esti- mated at about $10 \mathrm{~cm}^{2}$ in footprint, and $1 \mathrm{~kg}$ in mass, similar to the alpha proton $\mathrm{x}$-ray spectrometer $\left(\mathrm{APXS}^{9}\right)$ that was used in the 1997 Mars Pathfinder mission. However, unlike APXS, the thermionic source used in the AEXS is about $10^{4}$ times more intense $\left(6 \times 10^{13}\right.$ electrons/s for $10 \mu \mathrm{A}$ current compared to $2 \times 10^{9} \alpha$-particles/s for the APXS $50 \mathrm{mCi}$ source), resulting in much shorter spectrum acquisition time and less energy consumption (predicted $10 \mathrm{~s}$ and 50 $\mathrm{J} /$ spectrum compared to $10 \mathrm{~h}$ and $10 \mathrm{~kJ}$ for APXS). The spatial resolution, controlled by beam focusing and variable working distance from several hundred micrometers to about $1 \mathrm{~cm}$ diameter, is also much less that the $20 \mathrm{~cm}^{2}$ irradiated area for the APXS. These attributes will make it possible to bring the head of the instrument, containing the miniaturized electron gun and $\mathrm{x}$-ray detector, to a sample to perform in situ analysis rapidly on several spatial scales. Relative to an $\mathrm{XRF}$ instrument (which typically employs an $\mathrm{x}$-ray tube to provide the excitation $\mathrm{x}$ rays), the electron-induced fluorescence is a one-step process, using the primary electrons more efficiently (by a factor of about $10^{3}$ ) by directly exciting the characteristic $\mathrm{x}$ rays. Relative to a natural beta source, the thermionic emitter is much more intense, resulting in much shorter spectra acquisition time. Thus it is hoped that the use of a vacuum-isolation membrane will lead to the development of a low mass, low-volume, and low-energy consumption instrument suitable for rapid elemental analysis of surfaces in situ.

\section{ACKNOWLEDGMENTS}

The authors thank T. Van Zandt for suggesting the experiment, E. Hagen, W. Kelliher, T. Elam, and A. Kelly for useful discussions, and M. Buehler for help with detector calibration. S. Grannan and N. Bridges analyzed the use of the AEXS for Mars exploration. This work was supported by the NASA Cross Enterprise Technology Development and Planetary Instrument Definition and Development Programs, and by the Air Force office of Scientific Research.

${ }^{1}$ E. D. Green and G. S. Kino, J. Vac. Sci. Technol. B 9, 1557 (1991).

${ }^{2}$ A. Kelly, U.S. Patent No. 5,391,958 (21 Feb. 1995).

${ }^{3}$ T. George, "Pump-less microfabricated electron microscope," NASA New Technology Report NPO-20335, June 1997.

${ }^{4}$ G. D. Danilatos, Scanning 7, 1 (1985); Scanning Microsc. 4, 799 (1999).

${ }^{5}$ J. Z. Wilcox, "Simulation of transmissivity of electron beams in Mars atmosphere," JPL Internal Document, 1997.

${ }^{6}$ J. Feldman, J. Z. Wilcox, T. George, D. Barsic, and A. Scherer, 13th Lunar and Planetary Science Conference, 1999 (unpublished).

${ }^{7}$ D. Joy, Monte Carlo Modeling for Electron Microscopy and Microanalysis (Oxford University, New York, 1995).

${ }^{8}$ R. Browning, T. Eimori, E. P. Traut, B. Chui, and R. Pease, J. Vac. Sci. Technol. B 9, 3578 (1991).

${ }^{9}$ R. Reider, H. Vanke, T. Economou, and J. Turkevich, J. Geophys. Res. E 2, 102 (1997).

${ }^{10}$ B. L. Doyle, D. S. Walsh, and S. R. Lee, Nucl. Instrum. Methods Phys. Res. B 54, 244 (1991). 